\title{
Antitumor activity of the plant extract morin in tongue squamous cell carcinoma cells
}

\author{
YAWEN JI ${ }^{1,2}$, LINGLU JIA ${ }^{1,2}$, YUNPENG ZHANG ${ }^{1,2}$, YIXIAO XING ${ }^{1,2}$, XUAN WU $^{1,2}$, \\ BIN ZHAO ${ }^{1,2}$, DONDJIAO ZHANG ${ }^{1,2}$, XIN XU $^{1,2}$ and XU QIAO ${ }^{3}$ \\ ${ }^{1}$ Department of Oral Maxillofacial Surgery, School of Stomatology, Shandong University; \\ ${ }^{2}$ Department of Oral Maxillofacial Surgery, Shandong Provincial Key Laboratory of Oral Tissue Regeneration; \\ ${ }^{3}$ School of Control Science and Engineering, Shandong University, Jinan, Shandong 250012, P.R. China
}

Received March 17, 2018; Accepted August 7, 2018

DOI: $10.3892 /$ or.2018.6650

\begin{abstract}
Morin is a naturally occurring bioflavonoid originally isolated from members of the Moraceae family of flowering plants and it possesses antitumor activity in various human cancer cells. The present study explored the antitumor effects of morin in tongue squamous cell carcinoma (TSCC) cells in vitro and investigated the underlying molecular events. A TSCC cell line was treated with different doses of morin for up to $48 \mathrm{~h}$. Analyses of cell viability, using Cell Counting Kit-8 (CCK-8), EdU incorporation, colony formation, flow cytometric analysis of cell cycle distribution and apoptosis, wound healing assay, western blot analysis and qRT-PCR assays, were then performed. The data revealed that morin treatment reduced Cal27 cell proliferation and reduced the migration capacity of tumor cells in a dose-dependent manner. Morin treatment also significantly upregulated mammalian sterile 20-like 1 (MST1) and MOB kinase activator 1 (MOB1) phosphorylation in CAL27 cells, but suppressed nuclear translocation of yes-associated protein (YAP) through the induction of YAP phosphorylation in Cal27 cells. Moreover, the expression of YAP-targeting genes, such as CTGF, CYR61 and ANKRD, was downregulated in morin-treated TSCC cells, indicating that morin was able to activate the Hippo signaling pathway to inhibit YAP nuclear translocation and YAP-related transcriptional activity in TSCC cells. In conclusion, the data from the present study demonstrated that morin produces anti-TSCC activity in vitro through activation of the Hippo signaling pathway and the downstream suppression of
\end{abstract}

Correspondence to: Dr Xin Xu, Department of Oral Maxillofacial Surgery, School of Stomatology, Shandong University, 44-1 Wenhua Xi Road, Jinan, Shandong 250012, P.R. China

E-mail: xinxu@sdu.edu.cn

Dr Xu Qiao, School of Control Science and Engineering, Shandong University, 17923 Jingshi Road, Jinan, Shandong 250012, P.R. China E-mail: qiaoxu@sdu.edu.cn

Key words: tongue squamous cell carcinoma, morin, Hippo/YAP pathway, Yes-associated protein
YAP activity in TSCC cells. Future studies should assess the clinical antitumor effects of morin.

\section{Introduction}

Head and neck cancer remains as one of the most debilitating human neoplasms, and is the sixth most commonly diagnosed cancer worldwide. Histologically, 90\% of head and neck cancers are head and neck squamous cell carcinoma (HNSCC), and tongue squamous cell carcinoma (TSCC) contributes to a large proportion of HNSCC (1). In recent decades, advancements have been achieved in the conventional treatment of these cancers, including surgery, radiotherapy and chemotherapy. However, the prognosis of TSCC remains poor (2). Thus, a greater understanding of the pathogenesis of TSCC is needed in order to identify effective antitumor drugs that can be used to clinically control TSCC.

To this end, the present study focuses on a natural flavonoid that possesses anticancer effects. The potential chemotherapeutic effects of many flavonoids have been tested in different clinical trials (3). Morin is a naturally occurring bioflavonoid originally isolated from members of the Moraceae family of flowering plants. Morin can be isolated from amygdala ( $P$. guajava L.) as a yellow pigment (4,5). Morin exerts anticancer activity through the modulation of various gene-signaling pathways that are involved in cell proliferation and differentiation (6-10). Previous studies have also demonstrated that morin is able to induce apoptosis in prostate cancer, human leukemia HL-60 and multiple myeloma cells. Recently, studies have demonstrated that treatment with morin induces tumor cell apoptosis by activating the mitochondrial and caspase-3 pathway $(11,12)$.

The Hippo pathway is an important tumor suppressor pathway in various human cancers (13-18). The Hippo kinase cascade involves mammalian sterile 20-like 1/2 (MST1/2, also known as STK4/3), salvador (SAV1), large tumor suppressor 1/2 (LATS1/2), MOB kinase activator 1A/B (MOB1a/b) and Yes-associated protein (YAP) $(15,18,19)$. Activation of the Hippo kinase cascade leads to the phosphorylation of YAP and restriction of YAP translocsation into the cytoplasm for the induction of YAP degradation $(16,17)$. However, while the Hippo signaling cascade is inactive, YAP is translocated into 
the nucleus where it promotes the expression of various genes involved in cell proliferation and survival (15). In addition, MOB1 is an adapter protein and it can bind to upstream MST1; thus, it can regulate MST1 phosphorylation or bind to the downstream LATS1 protein to enable the trans-phosphorylation of YAP (14). The activity of the Hippo pathway regulates organ size during embryonic development in animals through the regulation of cell proliferation and apoptosis, and alteration of this pathway occurs in various types of human cancer $(13,20)$. Aberrant expression of the Hippo pathway proteins promotes the translocation of YAP into the nucleus and drives transcription of YAP-targeting genes and, therefore, enhances cancer cell proliferation and inhibits apoptosis (20-22). YAP can also stimulate epithelial-to-mesenchymal transition of tumor cells and drive the tumor-initiation capacity of cancer stem cells (23). To date, elevated YAP expression has been observed in many types of human cancers, including lung, liver, ovary, breast and colon cancers (24-28). Previous studies have demonstrated that morin was able to impede YAP nuclear translocation through the activation of Hippo signaling and foster apoptosis in human liver cancer HepG2 cells (29). Additionally, morin has demonstrated antitumor activity in human TSCC cells (6). In the present study, we further assessed whether morin treatment could regulate MST1 and MOB1 expression to constitutively activate the Hippo signaling pathway and inactivate YAP in TSCC cells in vitro. We determined the effect of morin treatment on the modulation of TSCC cell proliferation, apoptosis and migration and on the regulation of the phosphorylation of the protein components of the Hippo pathway and YAP target genes. We aimed to provide insightful information regarding the effect of morin on TSCC cells and to identify morin as a potential treatment strategy or preventative agent for TSCC.

\section{Materials and methods}

Cell line culture and drug treatment. The TSCC cell line, CAL27, was obtained from Shanghai Ninth People's Hospital (Shanghai, China) and cultured in a high-glucose Dulbecco's modified Eagle's medium (DMEM; HyClone Laboratories; GE Healthcare Life Sciences, Logan, UT, USA) supplemented with $10 \%$ fetal bovine serum (FBS; Gibco; Thermo Fisher Scientific, Inc., Waltham, MA, USA) with $5 \% \mathrm{CO}_{2}$ at $37^{\circ} \mathrm{C}$. For morin treatment, tumor cells were cultured in a complete cell culture medium supplemented with different doses of morin (0, 50, 100 and $150 \mu \mathrm{M}$; Yuanye Chemicals Co., Ltd., Shanghai, China), while dimethyl sulfoxide (DMSO; Beijing Solarbio Science \& Technology Co., Ltd., Beijing, China), the solvent, was used as the control treatment for up to 14 days.

Cell viability assay. Tumor cell viability was assayed following morin treatment using a cell viability Cell Counting Kit-8 (CCK-8; Dojindo Laboratories, Kumamoto, Japan). Briefly, Cal27 cells were seeded into 96-well plates at a density of 3,000 cells per well and grown overnight in a complete cell culture medium (see above) and then refreshed with morin-containing medium for 24 to $48 \mathrm{~h}$. At the end of each experiment, cell growth medium was added to $10 \mu \mathrm{l}$ of CCK-8 working solution and cells were further cultured for $90 \mathrm{~min}$ at $37^{\circ} \mathrm{C}$. The optical density value (OD) of the plates was then measured using a microplate reader at $450 \mathrm{~nm}$. The experiment was performed in triplicate and repeated at least three times.

Colony formation assay. Cal27 cells were plated into 6-well plates (1,000 cells/well) and grown overnight. Following this incubation, the cell culture medium was refreshed with morin-containing medium $(0,50,100$ and $150 \mu \mathrm{M})$ every 2 days for 14 days. At the end of the experiments, cells were washed with ice-cold phosphate-buffered saline (PBS), fixed using freshly made $4 \%$ polyformaldehyde/PBS solution and stained with $0.1 \%$ crystal violet. Cell colonies with more than 50 cells were counted under an inverted microscope (Olympus Corp., Tokyo, Japan). The experiment was performed in triplicate and repeated at least three times.

5-Ethynyl-2'-deoxyuridine (EdU) incorporation assay. We performed the EdU incorporation assay using an EdU kit from Guangzhou RiboBio Co., Ltd. (Guangzhou, China) according to the manufacturer's instructions. Specifically, Cal27 cells were plated into 24 -well plates at a density of 50,000 cells/well and grown overnight. The cells were then treated with different concentrations of morin $(0,50,100$ and $150 \mu \mathrm{M}$ ) for $24 \mathrm{~h}$. Following this, cell culture was added to $50 \mu \mathrm{l}$ of the EdU working solution and further cultured for $2 \mathrm{~h}$. At the end of the experiment, cells were washed with ice-cold PBS and fixed in $4 \%$ paraformaldehyde for $15 \mathrm{~min}$. The cells were then treated with $2 \mathrm{mg} / \mathrm{ml}$ glycine at room temperature for 5 min and stained with Apollo ${ }^{\circledR} 567$ and Hoechst working solution, in the dark, for $30 \mathrm{~min}$. Finally, the cell images were obtained using fluorescence microscopy (Olympus Corp.).

Wound-healing assay. Cal27 cells were grown and treated with various concentrations of morin $(0,50,100$ and $150 \mu \mathrm{M})$ for $24 \mathrm{~h}$. For the tumor cell wound-healing assay, the cells were tripsinized and reseeded into 6 -well plates at a density of $1 \times 10^{6}$ cells/well and grown overnight to reach $\sim 90 \%$ confluency. The cell monolayer was then scratched with a sterile $200-\mu 1$ pipette tip across the plates, washed with DMEM twice and cultured for up to $24 \mathrm{~h}$ in DMEM/0.1\% FBS as well as different the concentrations of morin. At each time-point $(0,6$ or $24 \mathrm{~h})$, the cell monolayers were photographed using an inverted microscope (Olympus Corp.). The wound healing areas were measured using ImageJ software 14.8 for Windows (National Institutes of Health, Bethesda, MD, USA). The experiment was performed in triplicate and repeated at least three times.

Flow cytometric Annexin V/PI apoptosis assay. Cal27 cells were first plated into 6 -well plates $\left(1 \times 10^{6}\right.$ cells/well $)$ and incubated overnight at $37^{\circ} \mathrm{C}$. Cells were then treated with different doses of morin $(0,50,100$ and $150 \mu \mathrm{M})$ for up to $48 \mathrm{~h}$. At the end of each experiment, both detached and attached cells were collected and incubated with the Annexin V-FITC/propidium iodide (PI) apoptosis detection kit (eBioscience, Vienna, Austria) according to the manufacturer's protocol. Specifically, $\sim 20,000$ cells were suspended in the binding buffer and $5 \mu \mathrm{l}$ of Annexin V-FITC was added. Cells were incubated for $10 \mathrm{~min}$, followed by a further 5-min incubation after the addition of $5 \mu \mathrm{l}$ of the PI staining solution at room temperature in the dark. 
Finally, the apoptosis level was measured using flow cytometry (FACSCalibur; BD Biosciences, San Jose, CA, USA).

Flow cytometric cell cycle assay. Cal27 cells were plated into 6-well plates $\left(1 \times 10^{6}\right.$ cells/well $)$ and incubated overnight. Cells were then treated with different doses of morin $(0,50$, 100 and $150 \mu \mathrm{M}$ ) for $24 \mathrm{~h}$. The cells were then harvested and washed with ice-cold PBS once and fixed in $70 \%$ ethanol at $4^{\circ} \mathrm{C}$ for $12 \mathrm{~h}$. On the next day, the cells were washed again with PBS and then stained with PI working solution at room temperature for $30 \mathrm{~min}$ in the dark. The cell cycle distribution of the cells was analyzed using flow cytometry (FACSCalibur; BD Biosciences).

Protein extraction and western blot analysis. Morin-treated Cal27 cells were lysed in radioimmunoprecipitation assay buffer (RIPA buffer; Beyotime Institute of Biotechnology, Shanghai, China) containing 1\% phenylmethylsulfonyl fluoride (PMSF; Beyotime Institute of Biotechnology) for $30 \mathrm{~min}$ on ice. Both cytoplasmic and nuclear extracts were then extracted using a nuclear fractionation protocol, following the manufacturer instructions (Beijing Solarbio Science \& Technology Co., Ltd.). The concentration of each protein sample was assayed using the bicinchoninic acid assay kit (Beijing Solarbio Science \& Technology) and equal amounts (20 $\mu \mathrm{g}$ of each) of protein samples were separated using $10 \%$ sodium dodecyl sulfate-polyacrylamide gel (Beyotime Institute of Biotechnology) electrophoresis and electrically transferred onto polyvinylidene fluoride (PVDF) membranes (Invitrogen; Thermo Fisher Scientific). For western blotting, the membranes were first incubated in 5\% non-fat milk at room temperature for $1 \mathrm{~h}$ and then incubated with a rabbit monoclonal anti-human YAP antibody (cat. no. 14074), a rabbit monoclonal anti-human phospho-YAP antibody (cat. no. 13619), a rabbit polyclonal anti-human MST1 antibody (cat. no. 3682), a rabbit polyclonal anti-human phospho-MST1 antibody (cat. no. 3681), a rabbit monoclonal anti-human MOB1 antibody (cat. no. 13730), and a rabbit monoclonal anti-human phospho-MOB1 antibody (cat. no. 8699) (all from Cell Signaling Technology, Inc., Danvers, MA, USA) at $4^{\circ} \mathrm{C}$ overnight. The next day, the membranes were washed with Tris-based saline-Tween-20 (TBS-T; $20 \mathrm{mmol} / 1$ Tris- $\mathrm{HCl}, 150 \mathrm{mmol} / 1 \mathrm{NaCl}$ and $0.05 \%$ Tween-20) three times for $10 \mathrm{~min}$ each time and then incubated in 1:5,000 HRP-conjugated goat anti-rabbit $\operatorname{IgG}$ (cat. no. 7074S; Cell Signaling Technology, Inc.). The protein bands were visualized using the Chemiluminescent HRP Substrate kit (EMD Millipore, Billerica, MA, USA). The level of protein expression was then normalized to that of GAPDH (cytoplasmic protein) and Histone H3 (nuclear protein).

Quantitative RT-PCR ( $q R T-P C R)$. The total cellular RNA was isolated from Cal27 cells that had been treated with morin for 24 h using a TRIzol ${ }^{\circledR}$ reagent (Takara Bio, Inc., Otsu, Japan). The RNA was then reversely transcribed into cDNA using a Reverse Transcriptase kit (Takara Bio) according to the manufacturer's protocols. These cDNA samples were then amplified with qPCR in $20 \mu$ of the reaction system, which contained the SYBR ${ }^{\circledR}$ Primix Ex Taq ${ }^{\mathrm{TM}}$ (Takara Bio), cDNA, each primer and RNase-free $\mathrm{H}_{2} \mathrm{O}$, following the manufacturer's protocol. Amplification conditions were set to an initial step of $95^{\circ} \mathrm{C}$ for

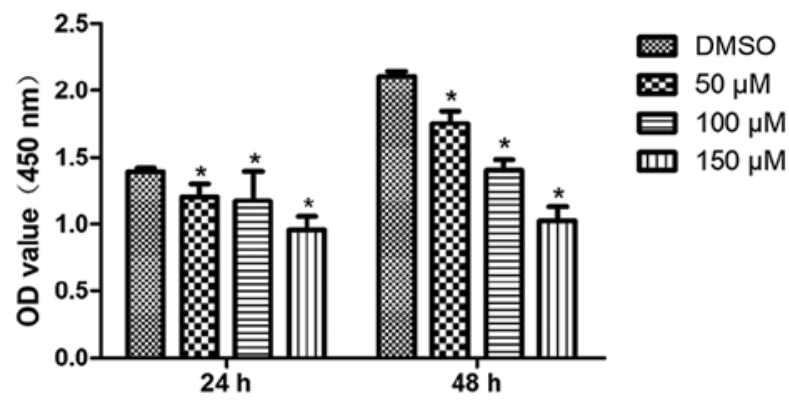

Figure 1. Effect of morin treatment on the reduction in TSCC cell viability. CCK-8 assay results demonstrated that morin treatment at 50, 100 and $150 \mu \mathrm{M}$ resulted in lower Cal27 cell viability throughout the duration of the experiment than was observed in the control group. This effect was dose-dependent; ${ }^{*} \mathrm{P}<0.05$, vs. the control (DMSO) group. TSCC, tongue squamous cell carcinoma.

$30 \mathrm{sec}$ and then 45 cycles of $95^{\circ} \mathrm{C}$ for $5 \mathrm{sec}, 60^{\circ} \mathrm{C}$ for $35 \mathrm{sec}$, and $72^{\circ} \mathrm{C}$ for $60 \mathrm{sec}$ and a final step at $40^{\circ} \mathrm{C}$ for $30 \mathrm{sec}$. The level of GAPDH mRNA was used as a control and the relative expression level of each RNA sample was calculated using the $2^{-\Delta \Delta \mathrm{Cq}}$ method (30). All experiments were performed in triplicate and repeated at least once. The primer sequences were GAPDH, 5'-GCACCGTCAAGGCTGAGAAC-3' and 5'-TGG TGAAGACGCCAGTGGA-3'; CTGF, 5'-TCTCCAACCTCT CCTACTAC-3' and 5'-GCACGTAGTTTCGATCACT3'; CYR61, 5'-CCTTGTGGACAGCCAGTGTA-3' and 5'-ACT TGGGCCGGTATTTCTTC-3'; and ANKRD, 5'-AGTAGA GGAACTGGTCACTGG-3' and 5'-TGGGCTAGAAGTGTC TTCAGAT-3'.

Statistical analysis. All data are summarized as the mean \pm standard error of the mean (SEM) of at least three replicates of each experiment and statistically analyzed using GraphPad Prism 5 (GraphPad Software Inc., La Jolla, CA, USA) with the two-tailed Student's t-test for two groups of data or the one-way analysis of variance (ANOVA) plus Tukey's post hoc test for multiple groups of data. $\mathrm{P}<0.05$ indicates statistical significance.

\section{Results}

Morin reduces TSCC cell proliferation. To evaluate the effects of morin on the regulation of TSCC cell proliferation, we performed cell viability (CCK-8), EdU incorporation and colony formation assays. Our CCK-8 assay data revealed that morin treatment significantly reduced tumor cell viability in a dose-dependent manner both at 24 and $48 \mathrm{~h}$ (Fig. 1). The results from the tumor cell colony formation assay also revealed that morin treatment significantly reduced the number of tumor cell colonies compared with the number of tumor cell colonies observed in the untreated control cells (Fig. 2). Moreover, morin treatment for $24 \mathrm{~h}$ led to a significantly lower percentage of EdU-positive cells than that observed in the control cells, in a dose-dependent manner (Fig. 3). These data indicated that morin inhibited TSCC cell proliferation.

Morin induces tumor cell cycle arrest and apoptosis. We further investigated how morin treatment inhibits tumor cell proliferation using flow cytometric apoptosis and cell cycle 

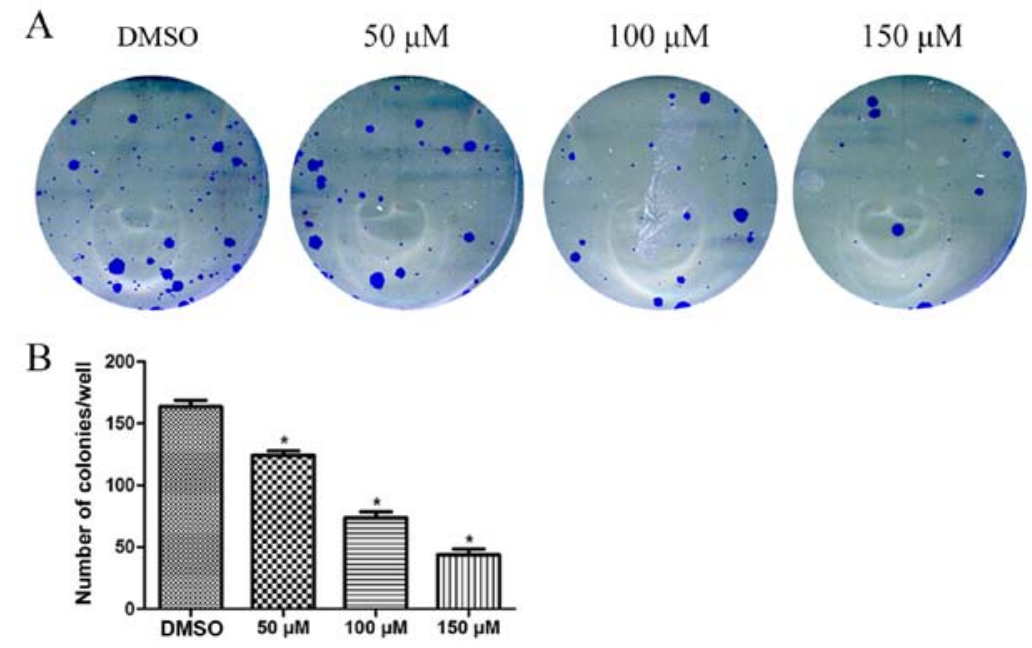

Figure 2. Effect of morin treatment on the reduction in TSCC colony formation. (A) The colony formation assay results revealed that morin treatment at 50, 100 and $150 \mu \mathrm{M}$ resulted in a lower number of tumor cell colonies than was observed in the DMSO group. This effect of morin was dose-dependent. (B) Quantification of the data from A. "P $<0.05$, vs. the control (DMSO) group. TSCC, tongue squamous cell carcinoma.

A

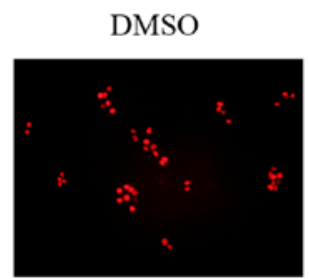

Hoechst
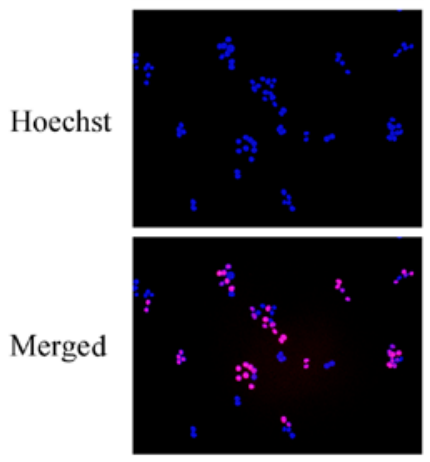

B

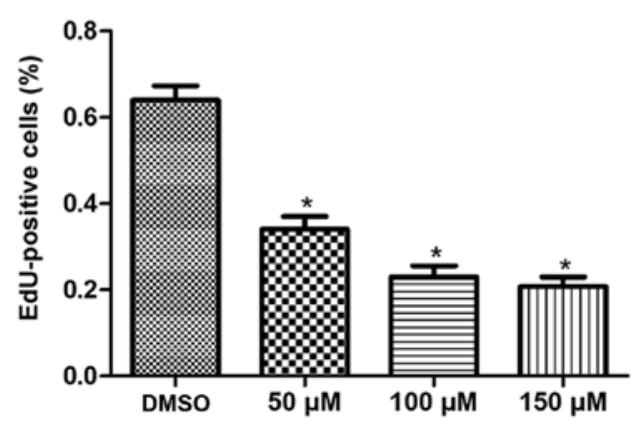

$50 \mu \mathrm{M}$
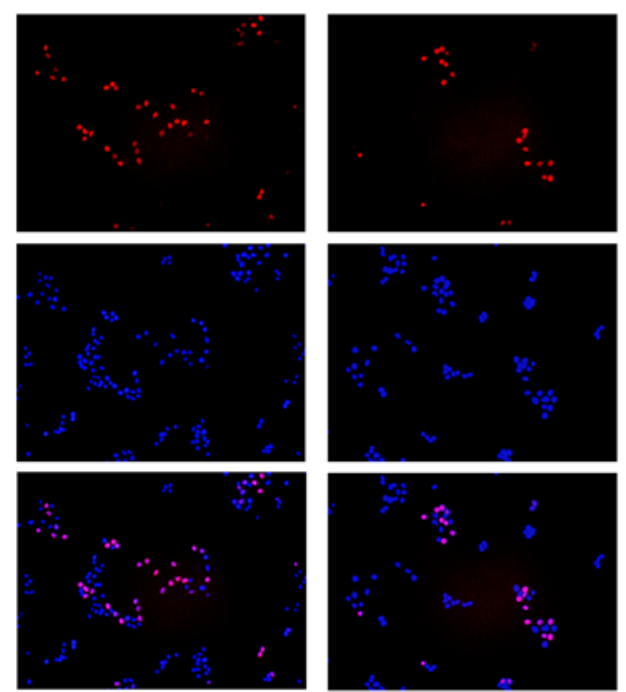

$100 \mu \mathrm{M}$
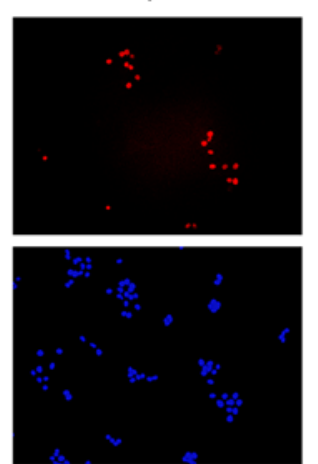

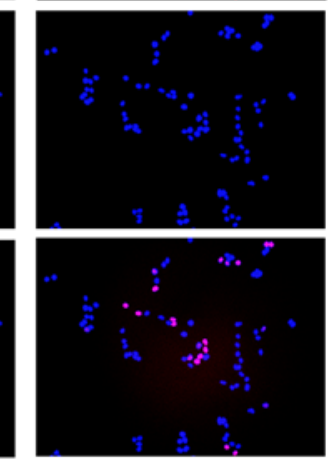

$150 \mu \mathrm{M}$

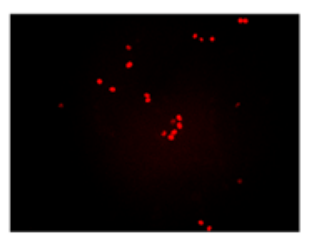

Figure 3. Effect of morin treatment on the reduction in TSCC cell EdU incorporation. (A) EdU incorporation assay. Cal27 cells were treated with morin at concentrations of 50,100 and $150 \mu \mathrm{M}$ and subjected to an EdU incorporation assay and counterstaining with Hoechst blue. The nuclei of EdU-positive cells are labeled red and all cell nuclei are labeled blue following Hoechst-blue staining (magnification, $\mathrm{x} 100$ ). (B) Quantification of the data from A. "P<0.05, vs. the (DMSO) control group. TSCC, tongue squamous cell carcinoma.

assays. Our data showed that morin treatment, for $24 \mathrm{~h}$, arrested tumor cells at the G1 phase of the cell cycle, and showed a significantly smaller percentage of $S$ phase cells than this percentage in the control group (Fig. 4A and B). Moreover, morin-treated tumor cells underwent apoptosis. Both the percentages of early and late apoptotic cells were significantly higher at $48 \mathrm{~h}$ of morin treatment at 100 and $150 \mu \mathrm{M}$ than in the control cells (Fig. 4C and D). 
A

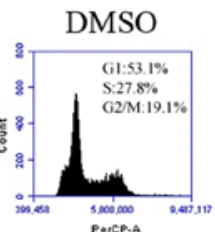

$\mathrm{C}$

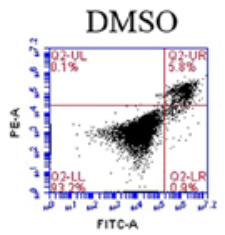

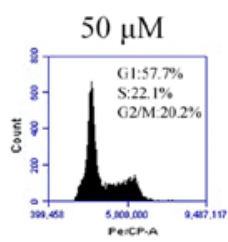
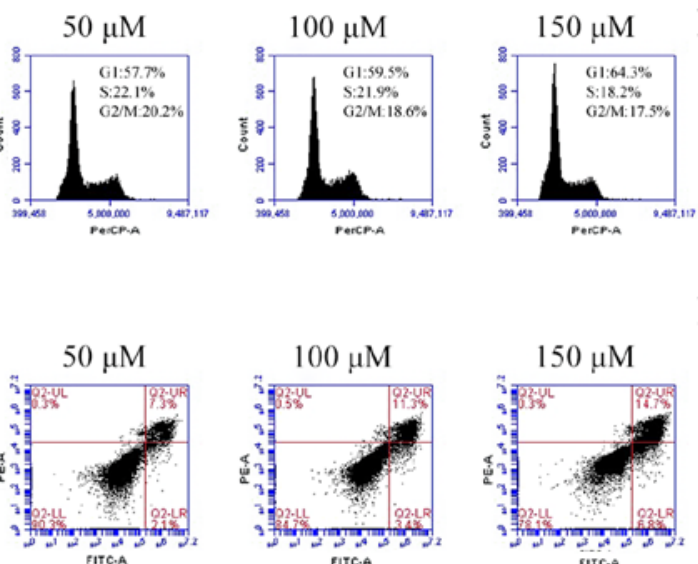

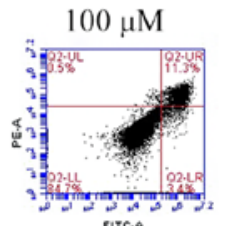

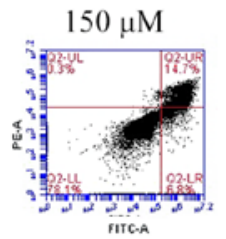

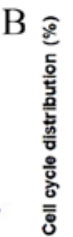

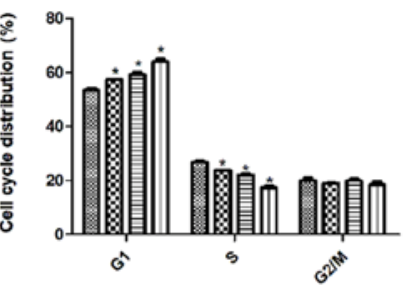

D
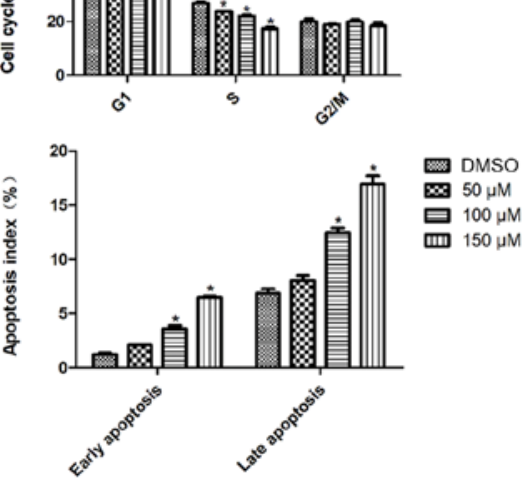

Figure 4. Morin induces tumor cell apoptosis and cell cycle arrest. (A) Representative plots of the cell cycle distribution of Cal27 cells after treatment of morin at concentrations of 50, 100 and $150 \mu \mathrm{M}$. (B) Statistical analysis of the flow cytometry results as described in A. (C) Flow cytometric apoptosis assay. Cal27 cells were treated with morin at concentrations of 50,100 and $150 \mu \mathrm{M}$ and subjected to the apoptosis assay. (D) Statistical analysis of the results from C; ${ }^{*} \mathrm{P}<0.05$, vs. the control (DMSO) group.

A

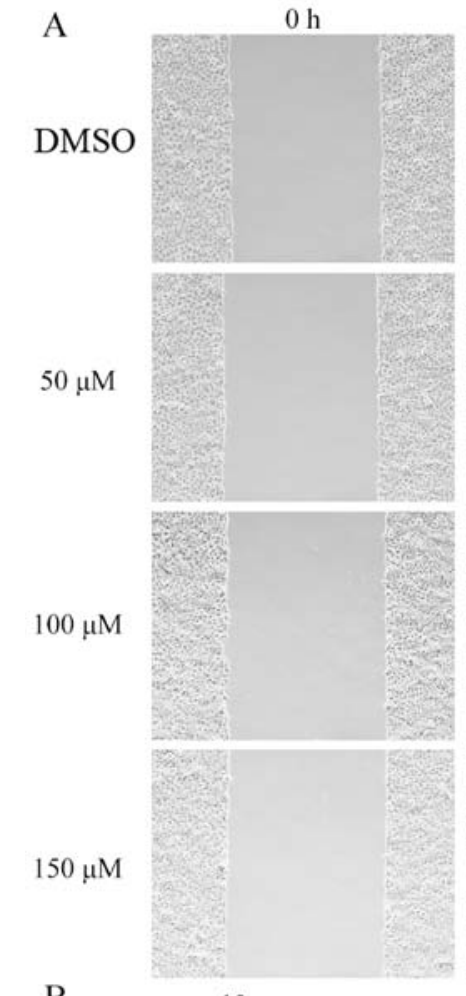

$50 \mu \mathrm{M}$

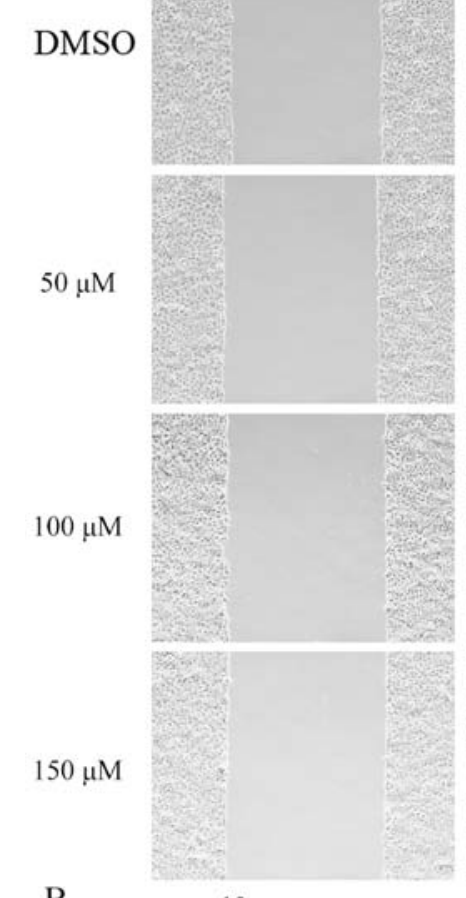

$100 \mu \mathrm{M}$

$150 \mu \mathrm{M}$

B
$6 \mathrm{~h}$
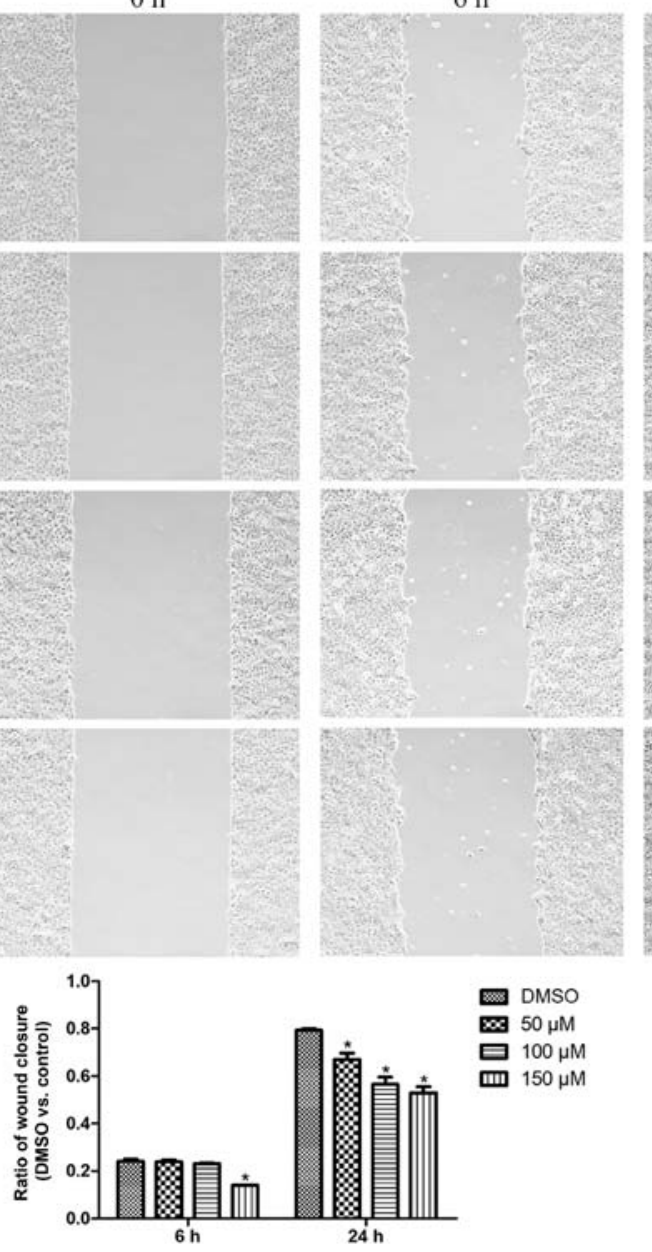

$24 \mathrm{~h}$
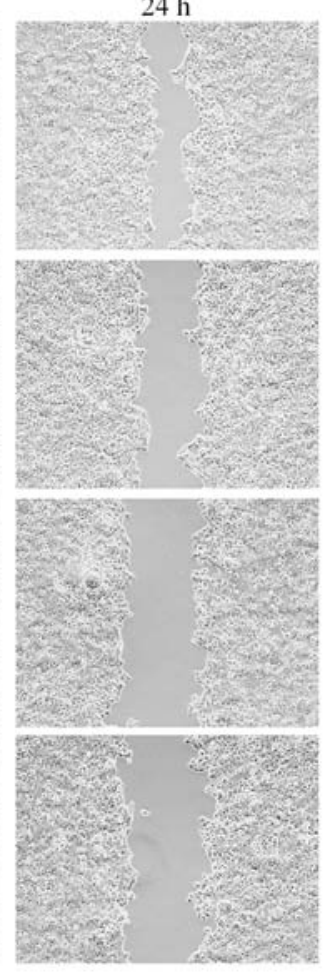
A

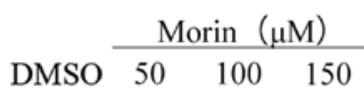

\section{MST1}

GAPDH

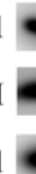

GAPDH

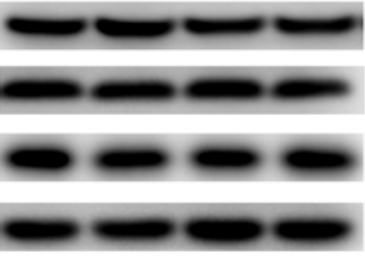

$$
\text { C }
$$$$
\text { DMSO } \begin{array}{ccc}
\multicolumn{2}{c}{\text { Morin }(\mu \mathrm{M})} \\
\cline { 2 - 4 } & 50 \quad 100 & 150
\end{array}
$$

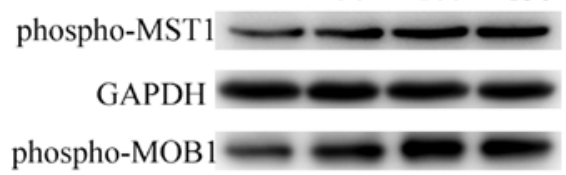

phospho-MOB1

GAPDH

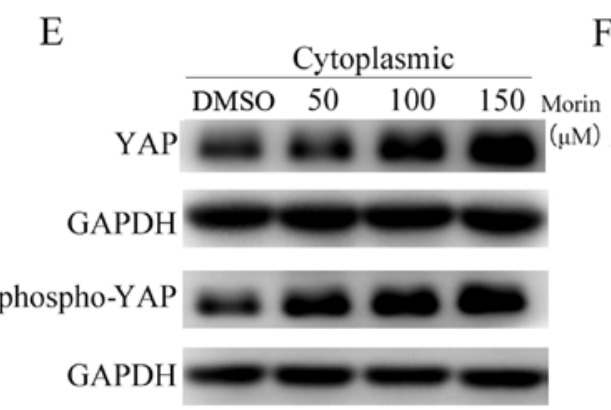

G

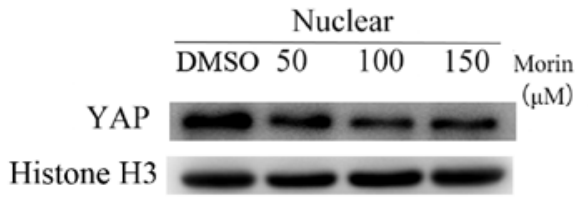

I

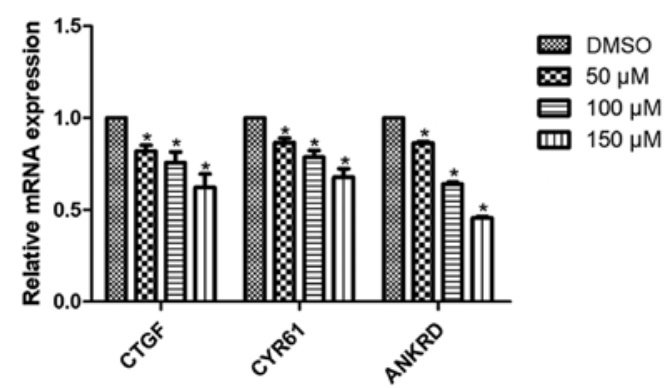

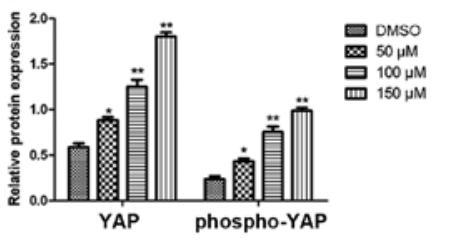

$\mathrm{H}$
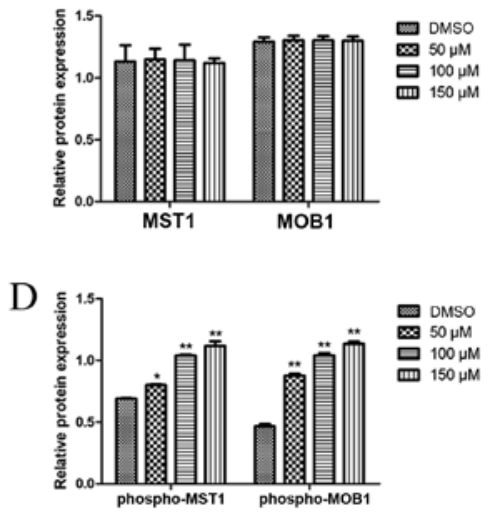

$\mathrm{F}$

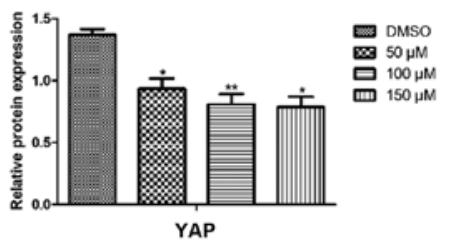

Figure 6. Effect of morin treatment on the regulation of gene expression. (A) Western blot analysis. The expression of MST1 and MOB1 proteins in Cal27 cells after $24 \mathrm{~h}$ of treatment with morin at concentrations of 50, 100 and $150 \mu \mathrm{M}$. (B) Quantification of the data from A. (C) Expression of phospho-MST1 and phospho-MOB1 proteins in Cal27 cells after $24 \mathrm{~h}$ of treatment with morin at concentrations of 50,100 and $150 \mu \mathrm{M}$. (D) Quantification of the data from C. (E) Expression of YAP and phospho-YAP in the cytoplasmic protein extracts from Cal27 cells after $24 \mathrm{~h}$ of treatment with morin at concentrations of 50, 100 and $150 \mu \mathrm{M}$. (F) Quantification of the data from E. (G) Expression of YAP in the nuclear protein extracts from Cal27 cells after $24 \mathrm{~h}$ of treatment with morin at concentrations of 50, 100 and $150 \mu \mathrm{M}$. (H) Quantification of the data from G. (I) YAP targeting genes were analyzed using qRT-PCR in Cal27 cells after $24 \mathrm{~h}$ of treatment with morin at concentrations of 50,100 and $150 \mu \mathrm{M} .{ }^{*} \mathrm{P}<0.05,{ }^{* *} \mathrm{P}<0.01$, vs. the control (DMSO) group.

wound healing capacity than the control cells, which showed quick closure of the wound (Fig. 5).

Morin activates Hippo pathway proteins and inhibits YAP nuclear translocation. Thus far, we demonstrated the antitumor activity of morin in TSCC cells in vitro. To further this study, we aimed to assess the underlying molecular pathways responsible for the action of morin on TSCC cells. Our western blot analysis revealed that the total levels of MST1 and MOB1 proteins were not significantly altered after morin treatment of TSCC cells (there was no significant difference between the results of the treated cells and the control cells; Fig. 6A and B). 
In contrast, the expression levels of phospho-MST1 and phospho-MOB1 proteins were significantly higher in morin-treated TSCC cells than these levels in the control cells (Fig. 6C and D). Furthermore, the cytoplasmic level of YAP protein was significantly upregulated in morin-treated TSCC cells and the ratio of phosphorylated YAP was also greater in the morin-treated tumor cells (Fig. 6E and F). In contrast, TSCC cells treated with high concentrations of morin exhibited a lower level of nuclear YAP protein than that of the control cells (Fig. 6G and H). We further investigated whether the changes in YAP protein localization that were observed following morin treatment correlated with an alteration in YAP-targeting proteins, such as CTGF, CYR61 and ANKRD. Our data showed that the expression of CTGF, CYR61 and ANKRD genes was significantly lower in the morin-treated Cal27 cells than that in the control cells (Fig. 6I). Taken together, our current data demonstrated that morin treatment upregulated the activity of the Hippo pathway but suppressed YAP nuclear translocation and YAP-related transcriptional activity in Cal27 cells.

\section{Discussion}

Morin displays wide-ranging pharmacological activities and low cytotoxicity against different types of human cancer cells (31). For example, previous studies have shown that morin possesses anti-liver cancer activity in the promotion stage of an in vivo liver carcinogenesis model (10). Additionally, morin has been shown to suppress breast cancer malignant behaviors through the inhibition of tumor cell epithelial-mesenchymal transition and Akt activation (32). Morin has also been suggested to induce apoptosis of human histiocytic lymphoma U937 cells through the upregulation of the Bcl-2-associated death promoter (BAD) protein levels (33). In tongue squamous cell carcinoma (TSCC), a recent study demonstrated that human TSCC cells were sensitive to morin-induced tumor cell growth inhibition (6). However, the underlying mechanism of morin in TSCC cells remains to be defined. Thus, the present study assessed the antitumor effect of morin in TSCC Cal27 cells and explored the underlying mechanisms. We found that morin treatment effectively decreased cell proliferation, colony formation, and migration of Cal27 cells in a dose-dependent manner. In the wound healing assay, cells were cultured for up to $24 \mathrm{~h}$. During this period of time cells do not proliferate to a great extent, thus this enabled us to avoid the effect of morin on cell proliferation in the wound healing assay. Our data showed that the wound healing capacity was inhibited to a greater extent in the morin-treated tumor cells than that observed in the control group, over a period of $24 \mathrm{~h}$. Additionally, the cell viability assay showed a significant reduction in tumor cell survival in the morin-treated group after a 24-h incubation period. Taken together, we have reason to believe that the wound assay will show a more significant difference between the drug treatment groups and the control group after 24 h. Furthermore, our cell cycle analysis showed that morin treatment led to cell cycle arrest at the G1 phase, with a significantly lower percentage of cells in the $S$ phase than that noted in the controls. Moreover, the data concerning the apoptosis analysis showed that morin treatment significantly increased the percentage of Cal27 cells that were undergoing early and late apoptosis. The morin-induced inhibition of TSCC cell growth and migration was demonstrated in our in vitro results.

Indeed, previous studies have demonstrated that different phytochemicals, including morin, possess antitumor activity in various types of human cancers (reviewed in ref. 4); morin has shown different pharmacological activities with very low cytotoxicity in humans (29); thus, in the present study, we treated TSCC Cal27 cells with up to $150 \mu \mathrm{M}$ morin, while a previous study of nude mouse melanoma cell xenografts treated the mice with $50 \mathrm{mg} / \mathrm{kg}$ of morin intraperitoneally (34). These doses could clinically be achievable (35). Previous studies have demonstrated that a low dose of morin reduced the cisplatin-induced toxicity of 293 cells and mouse kidney cells (36) and showed neuroprotective effect of morin in lead acetate-induced apoptosis in the rat brain (37). The induction of tumor cell apoptosis in response to morin at higher doses has been further confirmed in our current data.

Upon further exploration of the underlying mechanism of the morin antitumor activity in TSCC, we found that, at the gene level, morin upregulated the phosphorylation of the Hippo pathway proteins and inhibited YAP nuclear translocation. It is known that the Hippo pathway regulates cell growth, proliferation, apoptosis and organ size during embryonic development through alterations of the subcellular localization of YAP (38). For example, Song et al demonstrated that Hippo pathway-inactivated mice exhibited induced YAP phosphorylation with nuclear translocation, which led to the development of hepatocellular carcinoma (HCC) in mice (39). In contrast, re-activation of the Hippo pathway, in the HCC-derived cell line, promoted YAP phosphorylation and the suppression of HCC (39). Immunohistochemical studies of non-small cell lung cancer revealed that an elevated ratio of nuclear localization of YAP protein is associated with advanced tumor features and poor patient outcome (40-42). Furthermore, the level of YAP protein has been found to be upregulated in precancerous lesions in a rat model of liver cancer and upregulation of the nuclear level of YAP protein is frequently found in fully developed HCC (43). Elevated YAP expression and nuclear localization also occurs in mouse models of pancreatic cancer, and in tumors derived from human pancreatic adenocarcinoma cell lines $(44,45)$. The pancreas-specific YAP knockout inhibits tumor progression in a mouse pancreatic cancer model (46). In the present study, we also demonstrated that morin treatment was able to activate Hippo signaling through the upregulation of phospho-MST1 and phospho-MOB1 proteins. Additionally, YAP phosphorylation was induced by morin, and the level of YAP nuclear translocation and the expression of YAP-targeting CTGF, CYR61 and ANKRD genes were inhibited.

This study demonstrated that morin possesses antitumor activity in Cal27 cells through the activation of the Hippo pathway and suppression of YAP nuclear translocation in vitro. However, the use of only one cell line is a limitation of this study. Future study with more cell lines is needed to confirm our current data. In addition, we do know that morin, as a photochemical, should be able to target multiple gene or gene pathways, as is seen with other phytochemicals $(33,34)$. Thus, future studies will further explore the gene targets of morin.

In conclusion, the present proof-of-principle study demonstrated that morin treatment inhibited TSCC Cal27 cell 
proliferation and migration, but induced TSCC cell apoptosis and arrested tumor cells in the G1 phase of the cell cycle. At the gene level, morin treatment activated the Hippo pathway and inhibited YAP activity, indicating that morin possesses antitumor activity in TSCC in vitro.

\section{Acknowledgements}

The authors would like to thank the Shandong Provincial Key Laboratory of Oral Tissue Regeneration for their support of the present study.

\section{Funding}

The present study was supported in part by grants from the Shandong Provincial Natural Science Foundation (no. ZR2017MH031) and the Construction Engineering Special Fund of Taishan Scholars (no. ts201511106).

\section{Availability of data and materials}

The datasets used during the present study are available from the corresponding author upon reasonable request.

\section{Authors' contributions}

$\mathrm{XX}$ and $\mathrm{XQ}$ designed the study and the experiments. YJ and LJ conducted the experiments. YZ, YX, DZ, XW and BZ analyzed the data and YJ wrote the manuscript. All authors read and approved the manuscript and agree to be accountable for all aspects of the research in ensuring that the accuracy or integrity of any part of the work are appropriately investigated and resolved.

\section{Ethics approval and consent to participate}

Not applicable.

\section{Patient consent for publication}

Not applicable.

\section{Competing interests}

The authors declare that they have no competing interests.

\section{References}

1. Wang SS, Cen X, Liang XH and Tang YL: Macrophage migration inhibitory factor: A potential driver and biomarker for head and neck squamous cell carcinoma. Oncotarget 8: 10650-10661, 2017.

2. Kang H, Kiess A and Chung CH: Emerging biomarkers in head and neck cancer in the era of genomics. Nat Rev Clin Oncol 12: 11-26, 2015.

3. Birt DF and Bresnick E: Chemoprevention by nonnutrient components of vegetables and fruits. Springer US, 1991.

4. Aggarwal BB and Shishodia S: Molecular targets of dietary agents for prevention and therapy of cancer. Biochem Pharmacol 71: 1397-1421, 2006

5. Wijeratne SS, Abou-Zaid MM and Shahidi F: Antioxidant polyphenols in almond and its coproducts. J Agric Food Chem 54: 312-318, 2006.
6. Brown J, O'Prey J and Harrison PR: Enhanced sensitivity of human oral tumours to the flavonol, morin, during cancer progression: Involvement of the Akt and stress kinase pathways. Carcinogenesis 24: 171-177, 2003.

7. Sivaramakrishnan V and Niranjali DS: Morin regulates the expression of NF-kappaB-p65, COX-2 and matrix metalloproteinases in diethylnitrosamine induced rat hepatocellular carcinoma. Chem Biol Interact 180: 353-359, 2009.

8. Sivaramakrishnan V and Devaraj SN: Morin fosters apoptosis in experimental hepatocellular carcinogenesis model. Chem Biol Interact 183: 284-292, 2010.

9. Madankumar P, Naveenkumar P, Manikandan S, Devaraj H and Niranjalidevaraj S: Morin ameliorates chemically induced liver fibrosis in vivo and inhibits stellate cell proliferation in vitro by suppressing Wnt $/ \beta$-catenin signaling. Toxicol Appl Pharmacol 277: 210-220,2014.

10. MadankumarP,Naveenkumar P,DevarajHandNiranjalidevarajS: Morin, a dietary flavonoid, exhibits anti-fibrotic effect and induces apoptosis of activated hepatic stellate cells by suppressing canonical NF- $\kappa$ B signaling. Biochimie 110: 107-118, 2015.

11. Kuo HM, Chang LS, Lin YL, Lu HF, Yang JS, Lee JH and Chung JG: Morin inhibits the growth of human leukemia HL-60 cells via cell cycle arrest and induction of apoptosis through mitochondria dependent pathway. Anticancer Res 27: 395-405, 2007.

12. Gupta SC, Phromnoi K and Aggarwal BB: Morin inhibits STAT3 tyrosine 705 phosphorylation in tumor cells through activation of protein tyrosine phosphatase SHP1. Biochem Pharmacol 85: 898-912, 2013.

13. Saucedo LJ and Edgar BA: Filling out the Hippo pathway. Nat Rev Mol Cell Biol 8: 613-621, 2007.

14. Bichsel SJ, Tamaskovic R, Stegert MR and Hemmings BA: Mechanism of activation of NDR (nuclear Dbf2-related) protein kinase by the hMOB1 protein. J Biol Chem 279: 35228-35235, 2004.

15. Pan D: The hippo signaling pathway in development and cancer. Dev Cell 19: 491-505, 2010.

16. Oka T, Mazack V and Sudol M: Mst2 and Lats kinases regulate apoptotic function of yes kinase-associated protein (YAP). J Biol Chem 283: 27534-27546, 2008.

17. Huang J, Wu S, Barrera J, Matthews K and Pan D: The hippo signaling pathway coordinately regulates cell proliferation and apoptosis by inactivating Yorkie, the Drosophila homolog of YAP. Cell 122: 421-434, 2005.

18. Yu FX and Guan KL: The Hippo pathway: Regulators and regulations. Genes Dev 27: 355-371, 2013.

19. Halder G and Johnson RL: Hippo signaling: Growth control and beyond. Development 138: 9-22, 2011.

20. Zhao B, Tumaneng K and Guan KL: The Hippo pathway in organ size control, tissue regeneration and stem cell self-renewal. Nat Cell Biol 13: 877-883, 2011.

21. Zeng Q and Hong W: The emerging role of the hippo pathway in cell contact inhibition, organ size control, and cancer development in mammals. Cancer Cell 13: 188-192, 2008.

22. Yu FX, Zhao B and Guan KL: Hippo pathway in organ size control, tissue homeostasis, and cancer. Cell 163: 811-828, 2015.

23. Cordenonsi M, Zanconato F, Azzolin L, Forcato M, Rosato A, Frasson C, Inui M, Montagner M, Parenti AR, Poletti A, et al: The Hippo transducer TAZ confers cancer stem cell-related traits on breast cancer cells. Cell 147: 759-772, 2011.

24. Overholtzer M, Zhang J, Smolen GA, Muir B, Li W, Sgroi DC, Deng CX, Brugge JS and Haber DA: Transforming properties of YAP, a candidate oncogene on the chromosome 11q22 amplicon. Proc Natl Acad Sci USA 103: 12405-12410, 2006.

25. Steinhardt AA, Gayyed MF, Klein AP, Dong J, Maitra A, Pan D, Montgomery EA and Anders RA: Expression of Yes-associated protein in common solid tumors. Hum Pathol 39: 1582-1589, 2008.

26. Fernandez-L A, Northcott PA, Dalton J, Fraga C, Ellison D, Angers S, Taylor MD and Kenney AM: YAP1 is amplified and up-regulated in hedgehog-associated medulloblastomas and mediates Sonic hedgehog-driven neural precursor proliferation. Genes Dev 23: 2729-2741, 2009.

27. Song Y, Li L, Ou Y, Gao Z, Li E, Li X, Zhang W, Wang J, Xu L, Zhou Y, et al: Identification of genomic alterations in oesophageal squamous cell cancer. Nature 509: 91-95, 2014.

28. Mohseni M, Sun J, Lau A, Curtis S, Goldsmith J, Fox VL, Wei C, Frazier M, Samson O, Wong KK, et al: A genetic screen identifies an LKB1-MARK signalling axis controlling the Hippo-YAP pathway. Nat Cell Biol 16: 108-117, 2014. 
29. Perumal N, Perumal M, Kannan A, Subramani K, Halagowder D and Sivasithamparam N: Morin impedes Yap nuclear translocation and fosters apoptosis through suppression of Wnt $/ \beta$-catenin and NF- $\kappa$ B signaling in Mst1 overexpressed HepG2 cells. Exp Cell Res 15: 124-141, 2017.

30. Livak KJ and Schmittgen TD: Analysis of relative gene expression data using real-time quantitative PCR and the $2^{-\Delta \Delta C_{\mathrm{T}}}$ method. Methods 25: 402-408, 2001.

31. Gutiérrez RM, Mitchell S and Solis RV: Psidium guajava: A review of its traditional uses, phytochemistry and pharmacology. J Ethnopharmacol 117: 1-27, 2008.

32. Jin H, Lee WS, Eun SY, Jung JH, Park HS, Kim G, Choi YH, Ryu CH, Jung JM, Hong SC, et al: Morin, a flavonoid from Moraceae, suppresses growth and invasion of the highly metastatic breast cancer cell line MDA-MB-231 partly through suppression of the Akt pathway. Int J Oncol 45: 1629-1637, 2014.

33. Park C, Lee WS, Go SI, Nagappan A, Han MH, Hong SH, Kim GS, Kim GY, Kwon TK, Ryu CH, et al: Morin, a flavonoid from moraceae, induces apoptosis by induction of BAD protein in human leukemic cells. Int J Mol Sci 16: 645-659, 2014.

34. Hu J, Guo X and Yang L: Morin inhibits proliferation and self-renewal of $\mathrm{CD}_{133^{+}}$melanoma cells by upregulating miR-216a. J Pharmacol Sci 136: 114-120, 2018.

35. Hoque $\mathrm{A}$ and $\mathrm{Xu} \mathrm{XC}$ : Basic and translational research on dietary phytochemicals and cancer prevention. In: Targeting Cellular Signaling for Cancer Prevention and Therapy by Phytochemicals. pp127-156, 2013.

36. Singh MP, Chauhan AK and Sun CK: Morin hydrate ameliorates cisplatin-induced ER stress, inflammation and autophagy in HEK-293 cells and mice kidney via PARP-1 regulation. Int Immunopharmacol 56: 156-167, 2018.

37. Thangarajan $S$, Vedagiri A, Somasundaram S, Sakthimanogaran $R$ and Murugesan M: Neuroprotective effect of morin on lead acetate-induced apoptosis by preventing cytochrome $\mathrm{c}$ translocation via regulation of Bax/Bcl-2 ratio. Neurotoxicol Teratol 66 : 35-45, 2018.
38. Ling P, Lu TJ, Yuan CJ and Lai MD: Biosignaling of mammalian Ste20-related kinases. Cell Signal 20: 1237-1247, 2008.

39. Song H, Mak KK, Topol L, Yun K, Hu J, Garrett L, Chen Y, Park O, Chang J, Simpson RM, et al: Mammalian Mst1 and Mst2 kinases play essential roles in organ size control and tumor suppression. Proc Natl Acad Sci USA 107: 1431-1436, 2010.

40. Xie M, Zhang L, He CS, Hou JH, Lin SX, Hu ZH, Xu F and Zhao HY: Prognostic significance of TAZ expression in resected non-small cell lung cancer. J Thorac Oncol 7: 799-807, 2012.

41. Su LL, Ma WX, Yuan JF, Shao Y, Xiao W and Jiang SJ: Expression of Yes-associated protein in non-small cell lung cancer and its relationship with clinical pathological factors. Chin Med J 125: 4003-4008, 2012.

42. Noguchi S, Saito A, Horie M, Mikami Y, Suzuki HI, Morishita Y, Ohshima M, Abiko Y, Mattsson JS, König H, et al: An integrative analysis of the tumorigenic role of TAZ in human non-small cell lung cancer. Clin Cancer Res 20: 4660-4672, 2014.

43. Perra A, Kowalik MA, Ghiso E, Ledda-Columbano GM, Di Tommaso L, Angioni MM, Raschioni C, Testore E, Roncalli M, Giordano S, et al: YAP activation is an early event and a potential therapeutic target in liver cancer development. J Hepatol 61: 1088-1096, 2014.

44. Morvaridi S, Dhall D, Greene MI, Pandol SJ and Wang Q: Role of YAP and TAZ in pancreatic ductal adenocarcinoma and in stellate cells associated with cancer and chronic pancreatitis. Sci Rep 5: 16759, 2015.

45. Diep CH, Zucker KM, Hostetter G, Watanabe A, Hu C, Munoz RM, Von Hoff DD and Han H: Down-regulation of yes associated protein 1 expression reduces cell proliferation and clonogenicity of pancreatic cancer cells. PLoS One 7: e32783, 2012.

46. Zhang W, Nandakumar N, Shi Y, Manzano M, Smith A Graham G, Gupta S, Vietsch EE, Laughlin SZ, Wadhwa M, et al: Downstream of mutant KRAS, the transcription regulator YAP is essential for neoplastic progression to pancreatic ductal adenocarcinoma. Sci Signal 7: ra42, 2014. 